\title{
Right ventricular wall thickness indexed to body surface area as an echocardiographic predictor of acute pulmonary embolism in high-risk patients
}

\author{
Jerzy Wiliński', Anna Skwarek', Radosław Borek', Ositadima Chukwu², Katarzyna Ciuk², \\ Katarzyna Stolarz-Skrzypek ${ }^{3}$, Marek Rajzer ${ }^{3}$ \\ 'Department of Internal Medicine with Cardiology Subdivision, Blessed Marta Wiecka District Hospital, Bochnia, Poland \\ ${ }^{2}$ Faculty of Medicine, Jagiellonian University Medical College, Kraków, Poland \\ ${ }^{3} 1^{\text {st }}$ Department of Cardiology, Interventional Electrocardiology and Arterial Hypertension, Jagiellonian University Medical College, Kraków, Poland
}

Correspondence to: Jerzy Wiliński, MD, PhD, $1^{\text {st }}$ Department of Internal Medicine with Cardiology Unit, Blessed Marta Wiecka District Hospital,

Krakowska 31, 32-700 Bochnia, Poland,

phone: +48 1461533 17, e-mail: putamen@interia.pl Copyright by the Author(s), 2022 DOI: 10.33963/KP.a2021.0180

Received:

September 16, 2021

Accepted:

December 13, 2021

Early publication date:

December 14, 2021

\section{INTRODUCTION}

The abnormalities in acute pulmonary embolism (PE) observed in a transthoracic echocardiographic examination (TTE) are ascribed to the right ventricular (RV) pressure overload and dysfunction evoked by PE, but those findings can also be present in the absence of acute PE and may occur due to concomitant cardiac or respiratory diseases $[1,2]$. The recommendations for TTE use in PE diagnostics present a general approach without standard individualization to the patients' body size [1].

This study aimed to assess the usefulness of classic echocardiographic parameters indexed to the height and the body surface area (BSA) for prediction of acute PE in patients with a high clinical probability of PE referred for computed tomography pulmonary angiography (CTPA).

\section{METHODS}

\section{Study group}

This was a cross-sectional observational single-center study including consecutive patients referred for CTPA at an Internal Medicine Department with a high clinical probability of PE, from August 1, 2018 to June 11, 2019. The diagnostic algorithm and treatment regimens were adopted from the guidelines of the European Society of Cardiology [1, 3]. Exclusion criteria and echocardiographic assessment methodology are described in the Supplementary material. The study protocol complied with the Declaration of Helsinki and was approved by the Bioethics Committee of the Regional Medical Chamber in Tarnow, Poland (no. 3/0177/2019).

\section{Statistical analysis}

Statistical analysis was performed with the $\mathrm{R}$ Project for Statistical Computing version 3.6.3 (R Foundation for Statistical Computing, Free Software Foundation Inc., Vienna, Austria). Categorical variables were presented as absolute frequencies or relative percentages, while continuous variables as median with interquartile range (IQR) or as mean with standard deviation (SD). Categorical variables were compared using the $\mathrm{x} 2$ test or the Fisher test.

The normality of distribution was investigated with the Shapiro-Wilk test. For quantitative variables and normal distribution, the t-test was applied. For the non-normal distribution, the Mann-Whitney test was used. Two-sided $P$-values $<0.05$ were considered statistically significant. Calculated $P$-values were not adjusted for multiple testing. Detailed statistical analysis is described in the Supplementary material.

\section{RESULTS AND DISCUSSION}

The study comprised 108 consecutive patients, 5 of whom had contraindications to CTPA and were transferred to another unit for further diagnostics. Another 9 patients had echocardiograms of poor quality that were excluded. Three patients had nondiagnostic CTPA. Finally, 91 individuals were eligible to 
be enrolled in the survey. Baseline characteristics of these patients are presented in the Supplementary material, Table S1. As many as 49 patients had PE confirmed in CTPA; 22 subjects had central PE (44.9\%), whereas 27 individuals (55.1\%) had peripheral PE: segmental -18 (36.7\%) or subsegmental - 9 (18.4\%). Forty-two patients had no radiological signs of $\mathrm{PE}$.

\section{Clinical and biochemical variables}

Considering clinical data, there were no differences in sex, age, body size, clinical prediction rules for $\mathrm{PE}$, or in most comorbidities. The individuals with PE had less often coronary artery disease (CAD) and chronic heart failure (CHF, Supplementary material, Table S1). In the analysis of laboratory tests results, they had only a higher concentration of D-dimer (Supplementary material, Table S2). The calculated cut-off value for D-dimer as a single predictor of $\mathrm{PE}$, with a sensitivity of $57 \%$ and specificity of $80 \%$ in the study group, was $4.891 \mathrm{ng} / \mathrm{ml}$.

\section{Classic TTE parameters}

The only parameter that was different in the groups of patients with and without PE was the right ventricular wall thickness (RVWT, average values of 4.97 [0.77] vs. 5.58 [1.75] $\mathrm{mm} ; P=0.046$ ) (Supplementary material, Table S3). There were no significant differences regarding the severity of valvular defects between the groups.

\section{Indexation of echocardiographic parameters to height}

The left atrial end-diastolic area (LA EDA) indexed to height and RVWT indexed to height were significantly lower in the group with PE (Supplementary material, Table S1).

\section{Indexation of echocardiographic parameters to BSA}

Indexation to BSA revealed the differences between patients with and without PE in the left ventricle (LV) and the left atrium (LA) sizes. The indexed derivatives of left ventricular end-diastolic diameter (LVEDd), the left ventricular transverse diameter (LVTD), the left atrium diameter parasternal window - long-axis views (LAd PLAX), LA EDA - were decreased in the group with PE. RVWT indexed to BSA was also lower in patients with PE (Supplementary material, Table S3).

\section{Specific echocardiographic signs of PE}

The only specific TTE sign for PE, i.e. a mobile thrombus in the right atrium or right ventricle, was more frequently observed in the PE group (Supplementary material, Table S4).

\section{Clinical and echocardiographic predictors for PE}

In the univariate logistic regression the variables significantly associated with the PE diagnosis comprised (1) CAD, $\mathrm{CHF}$ (occurrence of both lowered the chances for PE); (2) serum $\mathrm{D}$-dimer concentration (positively correlated with $\mathrm{PE}$ );
(3) RVWT; (4) LAd PLAX indexed to BSA; (5) LVEDd indexed to $B S A$; (6) pulmonary artery (PA) indexed to BSA; (7) LA EDA indexed to BSA; (8) RVWT indexed to BSA (all negatively correlated with $\mathrm{PE}$ ). In the multivariable analysis, the factors independently positively associated with PE included the absence of CAD, higher D-dimer concentration, and lower RVWT indexed to BSA (Supplementary material, Table S5).

\section{Clinical significance}

To evaluate a possible clinical utility of RVWT indexed to BSA, we performed receiver-operating characteristic (ROC) analysis and calculated the optimal cut-off value. The obtained area under the curve (AUC) was $0.68(95 \%$ confidence interval $[\mathrm{Cl}], 0.56-0.80 ; P=0.002)$, the upper cut-off value of $3.19 \mathrm{~mm} / \mathrm{m}^{2}$ with accuracy of $60 \%$ predicted acute PE. Its sensitivity was $0.14(95 \% \mathrm{Cl}, 0.06-0.27)$ and specificity was $0.69(95 \% \mathrm{Cl}, 0.53-0.82$; Supplementary material, Figure S1). To enhance the predictive potential of RVWT indexed to BSA, we combined it with D-dimer levels in the multiple regression model. ROC and model statistics are shown in the Supplementary material, Figure S2. In this model AUC equaled $0.776(95 \% \mathrm{Cl}, 0.669-0.883 ; P<0.001)$, with sensitivity of $0.76(95 \% \mathrm{Cl}, 0.60-0.89)$ and specificity of 0.68 (95\% Cl, 0.50-0.82).

Echocardiography is an underutilized tool in PE diagnostics and outcome prognosis. RV dysfunction on TTE is associated with an increased risk of short-term mortality even in an initially hemodynamically stable patient with PE [4-6]. TTE may also help to identify possible candidates for early thrombolytic treatment, thus TTE should be widely used in clinical practice in all patients with PE $[7,8]$. In the presented study, nearly $50 \%$ of subjects had a negative result for PE in CTPA. It stresses the urgent need for the search for additional predictive factors. Our results of RVWT indexed to BSA confirm the usefulness of TTE and its potential in this area.

Indexing of heart cavities size to BSA is becoming a standard in echocardiography [9]. Nevertheless, indexation of ventricular wall thickness (WT) to BSA has an emerging role. Left ventricular wall thickness (LVWT) showed significant relation with BSA. Indexed LVWT was similar in men and women, but it differed among races and was associated with decreased hypertrophic cardiomyopathy diagnoses [10-12].

The only biochemical and clinical variables independently associated with PE diagnosis were D-dimer concentration and CAD. The current algorithms use D-dimer testing in patients with low/moderate clinical probability of $P E$ to rule out $P E$ [1]. Our findings should stimulate future research on D-dimer in PE diagnostics.

Due to low accuracy and sensitivity, RVWT indexed to BSA is not useful as a single indicator of PE. An open question remains about possible associations between RVWT and other RV indices and their diagnostic utility for PE diagnosis, which is beyond the scope of this article but requires future research. Standard indexation of TTE param- 
eters reveals differences between the groups of patients of high clinical probability of PE with and without acute PE. The factors independently associated with PE include the absence of CAD, higher D-dimer concentration, and lower RVWT indexed to BSA.

\section{Supplementary material}

Supplementary material is available at https://journals. viamedica.pl/kardiologia_polska.

\section{Article information}

Conflict of interest: None declared.

Open access: This article is available in open access under Creative Common Attribution-Non-Commercial-No Derivatives 4.0 International (CC BY-NC-ND 4.0) license, allowing to download articles and share them with others as long as they credit the authors and the publisher, but without permission to change them in any way or use them commercially. For commercial use, please contact the journal office at kardiologiapolska@ptkardio.pl.

\section{REFERENCES}

1. Konstantinides SV, Meyer G, Becattini C, et al.2019 ESC Guidelines for the diagnosis and management of acute pulmonary embolism developed in collaboration with the European Respiratory Society (ERS): The Task Force for the diagnosis and management of acute pulmonary embolism of the European Society of Cardiology (ESC). Eur Heart J. 2020; 41(4): 543-603, doi: doi.org/10.1093/eurheartj/ehz405.

2. Bova C, Greco F, Misuraca G, et al. Diagnostic utility of echocardiography in patients with suspected pulmonary embolism. Am J Emerg Med. 2003; 21(3): 180-183, doi: 10.1016/s0735-6757(02)42257-7, indexed in Pubmed: 12811708.

3. Konstantinides SV, Torbicki A, Agnelli G, et al. Guidelines on diagnosis and management of acute pulmonary embolism. Eur Heart J. 2014; 35(43): 3033-3069, doi: 10.1093/eurheartj/ehu283, indexed in Pubmed: 25173341 [published correction appears in Eur Heart J. 2015; 36(39): 2666].
4. Sanchez O, Trinquart L, Colombet I, et al. Prognostic value of right ventricular dysfunction in patients with haemodynamically stable pulmonary embolism: a systematic review. Eur Heart J. 2008; 29(12): 1569-1577, doi: 10.1093/eurheartj/ehn208, indexed in Pubmed: 18495689.

5. Coutance G, Cauderlier E, Ehtisham J, et al. The prognostic value of markers of right ventricular dysfunction in pulmonary embolism: a meta-analysis. Crit Care. 2011; 15(2): R103, doi: 10.1186/cc10119, indexed in Pubmed: 21443777.

6. Barco S, Mahmoudpour SH, Planquette B, et al. Prognostic value of right ventricular dysfunction or elevated cardiac biomarkers in patients with low-risk pulmonary embolism: a systematic review and meta-analysis. Eur Heart J. 2019; 40(11): 902-910, doi: 10.1093/eurheartj/ehy873, indexed in Pubmed: 30590531

7. Konstantinides S. Pulmonary embolism: impact of right ventricular dysfunction. Curr Opin Cardiol. 2005; 20(6): 496-501, doi: 10.1097/01. hco.0000179818.65329.bb, indexed in Pubmed: 16234620.

8. Sławek-Szmyt S, Jankiewicz S, Smukowska-Gorynia A, et al. Implementation of a regional multidisciplinary pulmonary embolism response team: PERT-POZ initial 1-year experience. Kardiol Pol. 2020; 78(4):300-310, doi: 10.33963/KP.15230, indexed in Pubmed: 32165606.

9. Rudski LG, Lai WW, Afilalo J, et al. Guidelines for the echocardiographic assessment of the right heart in adults: a report from the American Society of Echocardiography endorsed by the European Association of Echocardiography, a registered branch of the European Society of Cardiology, and the Canadian Society of Echocardiography. J Am Soc Echocardiogr. 2010; 23(7): 685-788, doi: 10.1016/j.echo.2010.05.010, indexed in Pubmed: 20620859

10. Sheikhsaraf B, Allah NH, Ali AM. Association of body surface area and body composition with heart structural characteristics of female swimmers. Int J Exerc Sci. 2010; 3(3): 97-101, indexed in Pubmed: 27182334.

11. LaBounty TM, Bach DS, Bossone $E$, et al. Effect of race on echocardiographic measures of cardiac structure and function. Am J Cardiol. 2019; 124(5):812-818, doi: 10.1016/j.amjcard.2019.05.049, indexed in Pubmed: 31296366.

12. Huurman R, Schinkel AFL, van der Velde $\mathrm{N}$, et al. Effect of body surface area and gender on wall thickness thresholds in hypertrophic cardiomyopathy. Neth Heart J.2020;28(1):37-43, doi: 10.1007/s12471-019-01349-1, indexed in Pubmed: 31776912. 